(C) Springer-Verlag 1991

\title{
Hallux valgus avec ou sans métatarsalgies
}

\section{GECO, Janvier 1990}

\author{
Gérard Copin ${ }^{1}$ et Christian Debaëne ${ }^{2}$ \\ ${ }^{1}$ CTO, avenue Achille-Baumann, BP 96, F-67400 Illkirch-Graffenstaden, France \\ ${ }^{2}$ Hôpital de Wissembourg, F-67160 Wissembourg, France
}

Plus de mille dossiers d'hallux valgus opérés ont été revus par les membres permanents du GEGO pour la $14 \mathrm{e}$ Réunion Elargie qui s'est tenue aux Arcs, en janvier 1990.

L'étude informatique a été réalisée à Strasbourg pour 846 dossiers et à Dijon pour les 177 autres.

Le but de ce travail était de pouvoir comparer les résultats des différentes techniques opératoires utilisées et de dégager une indication opératoire, compte tenu de l'importance de la déformation initiale de l'hallux valgus.

Après un rappel de la physiopathogénie de l'hallux valgus, par Max Perrin, la symptomatologie clinique de la série étudiée a été présentée par Christian Debaëne, alors que l'aspect radiographique était évidemment du ressort de Jean-Claude Dosch.

Les différentes techniques opératoires ont été regroupées par Gérard Copin, en cinq chapitres, en rappelant leur conception historique et les modalités opératoires :

1. La plus ancienne technique est l'exostosectomie métatarsienne de Schede.

2. Les opérations sur les parties molles comprennent :

- le recentrage isolé de la sangle sésamoïdienne décrit par Keller, en 1904, repris par Morton, en 1935 et diffusé en France par Lelièvre, dans son livre en 1961,

- la libération externe, métatarsophalangienne avec ténotomie de l'abducteur, décrite en 1875 par Petersen et réintroduite en France par Roy-Camille en 1962,

- la transposition ténodèse de l'abducteur, décrite en 1928 par Mac Bride et diffusée en France par Meary.

3. Les procédés de réaxation osseuse comprennent :

- l'ostéotomie de la phalange proximale P1 décrite par Giannestras et diffusée en France par Gauthier,

- l'ostéotomie de la base métatarsienne M1 très en vogue dans les services de Groulier à Marseille et de Delagoutte à Nancy, pouvant associer les deux ostéotomies au niveau de M1 et $\mathrm{P} 1$ au cours de la même intervention,

- l'ostéotomie sous-capitale de M1 décrite par Hohmann, en 1921,

- l'ostéotomie bipolaire de M1, décrite par Schnepp et Carret à Lyon.

4. Les arthroplasties métatarso-phalangiennes du gros orteil commprennent :

- la classique opération de KellerBrandes avec résection de la base de $\mathrm{P} 1$, associée au recentrage de la sangle sésamoïdienne selon Lelièvre, sans oublier la fixation des sésamoïdes au tendon fléchisseur et au moignon de P1, pour éviter leur recul, selon Antonio Viladot,

- la réimplantation ostéocartilagineuse de la base de P1 décrite par Regnauld à Nantes,

- l'arthroplastie par implant sylastic de Swanson, métatarso-phalangienne, proposée en 1973 par Michon, Delagoutte et Jandeaux, parallèlement à l'utilisation d'un implant sylastic de la base de P1, type bouchon.

5. L'arthrodèse métatarso-phalangienne qui pérénise la réduction des grosses déformations, tout en laissant persister une fonction satisfaisante par la mobilité interphalangienne.

Dans cette étude, il a été tenu compte de la préférence des uns et des autres pour une technique particulière, en leur confiant l'analyse des cas opérés selon cette technique.

Il est à noter que la population d'hallux valgus est à peu près semblable dans chaque série des cas opérés selon une technique opératoire, en tenant compte de l'âge, de l'importance, de la déformation et des lésions associés.

20 cas d'exostosectomie et recentrage de la sangle sésamoïdienne ont été analysés par Christian Debaëne, alors que les 142 recentrages de la sangle sésamoïdienne, avec libération dorsale des orteils, ont été présentés par Max Perrin qui les avait opérés.

Patrice Diebold a revu les 113 cas d'opération de Petersen, réalisés à Nancy.

Jean Berger, avec une étude spéciale de l'hallux valgus de l'enfant, a analysé les 36 cas d'opération de Mac Bride chez l'adulte.

Jean-Claude Schwartz s'est intéressé aux 66 cas d'ostéotomie basale métatarsienne $\mathrm{M} 1$ et aux 34 ostéotomies phalangiennes de $\mathrm{P} 1$ réalisées à Colmar. 
Les 284 cas d'ostéotomie souscapitale de M1, selon Hohmann, ont été analysés par Gérard Copin.

Les 102 cas de résection arthroplastique selon Keller-Brandes ont été revus par Jean-Claude Steib.

Les 104 cas d'implants métatarsophalangiens de Swanson ont été présentés par Michel Jandeaux, qui les avait mis en place.

Philippe Segal a revu les 50 cas d'arthrodèse métatarso-phalangienne du gros orteil.

Dans la grande majorité des cas, les métatarsalgies font partie du tableau clinique de l'hallux valgus par insuffisance fonctionnelle d'appui du premier rayon. Elles sont améliorés par la cure de l'hallux valgus. Toutefois, dans certains cas, les métatarsalgies persistent et ont motivé secondairement une réintervention par ostéotomie avec résection au niveau de la base des métatarsiens médians M2, M3, M4, permettant le relèvement et le recul des têtes métatarsiennes. Une courte série d'association de cette ostéotomie à l'opération de Hohmann est présentée par Gérard Copin.
Enfin, Jacques Xenard, après avoir étudié les suites post-opératoires de toutes ces interventions, a rappelé les modalités de rééducation fonctionnelle et d'appareillage de ces pieds opérés.

Les résultats globaux ont montré que la cure de l'hallux valgus était toujours bénéfique, mais que les résultats dépendaient de l'importance de l'hallux valgus initial et des techniques opératoires.

Compte tenu de ces éléments, les indications opératoires suivantes peuvent être proposées :

- 1'hallux valgus débutant, mais douloureux, dont la déformation métatarso-phalangienne est inférieure à $30^{\circ}$, est du domaine de l'opération de Mac Bride ou de Petersen, tout en se méfiant des risques d'hypercorrection de ces interventions,

- l'hallux valgus habituel de 30 à $45^{\circ}$ bénéficera au mieux des techniques d'ostéotomie métatarsienne, soit de la base associée à un recentrage de la sangle sésamoïdienne et d'une exostosectomie, soit du col métatarsien extra- articulaire selon Hohmann, permettant d'obtenir la meilleure force d'appui de la pulpe du gros orteil,

- l'hallux valgus, très important, supérieur à $50^{\circ}$, sera au mieux stabilisé par une arthrodèse métatarso-phalangienne, bien que l'arthroplastie de Swanson soit concurentielle. Mais celle-ci est entachée de critiques biologiques d'intolérance au sylastic, qui toutefois, n'a pas été constatée dans la série présente,

- nous ne pouvons plus conseiller l'opération de Keller-Brandes, même chez la personne âgée, qui bénéficiera au mieux de l'arthrodèse métatarsophalangienne, ni la remise en tension isolée de la sangle sésamoïdienne, selon Lelièvre, bien que l'un de nous, Max Perrin, en soit satisfait, à condition d'y associer la libération dorsale des orteils corrigeant l'enclavement métatarsien.

Ainsi, devant le dédale de la multiplicité des techniques opératoires, nous nous sommes donnés un fil d'Ariane pour traiter au mieux chaque cas particulier d'hallux valgus. 